\title{
A Two-Dimensional Architecture for End-to-end Resource Management in Virtual Network Environments
}

\author{
Ning Wang ${ }^{1}$, Yan Zhang ${ }^{2}$, Joan Serrat ${ }^{3}$, Juan Luis Gorricho ${ }^{3}$, Tao Guo ${ }^{1}$, Zheng Hu ${ }^{4}$, Ping Zhang ${ }^{4}$ \\ ${ }^{1}$ University of Surrey, U.K. \\ ${ }^{2}$ Simula Research Laboratory, Norway \\ ${ }^{3}$ Universitat Politècnica de Catalunya Spain \\ ${ }^{4}$ Beijing University of Post and Telecommunications, P. R. China
}

\begin{abstract}
In recent years, various network virtualisation techniques have been proposed for flexibly supporting heterogeneous services over virtual network platforms. However, systematic views on how virtual network resources (VNRs) can be practically managed in such open environments has been missing till now. To fill the gap, we present in this article a two-dimensional architecture for end-to-end VNR management from distinct viewpoints of service providers (SPS) and network providers (NPs). The horizontal dimension of VNR management allows SPs to bind VNRs rented from heterogeneous NPs to form unified end-to-end service delivery platforms. The vertical dimension of VNR management enables NPs to perform cost-efficient allocation of VNRs to requesting SPs, but without necessarily forcing themselves to collaborate with each other. Such a VNR management architecture will complement existing network virtualisation platforms in accelerating the realisation of virtual resource sharing in the future Internet business marketplaces.
\end{abstract}

\section{Introduction}

Network virtualisation has been extensively investigated in the recent years, as it is regarded as a promising solution to enabling flexible utilisation of network resources. The rationale behind such a paradigm is the newly evolved business relationship between stakeholders, with the separation of various service providers (SPs) from the underlying network (infrastructure) provider (NP) [1]. Specifically, SPs offer a wide variety of networked services on top of the Internet, but do not necessarily own any physical network resources by themselves. On the other hand, NPs may logically partition their physical network resources into virtual resource slices (also known as substrates [2] or planes [3]) and lease them to various requesting SPs according to their distinct service requirements. As such, the key advantage of network virtualisation is the high flexibility in resource sharing between heterogeneous networked services on top of a common physical network infrastructure. In addition, network virtualisation is able to alleviate the technical challenges in providing end-to-end services across multiple domains, without being constrained by the business/operational tussles between autonomous NPs. This is because the provisioning of inter-domain virtual networks does not force compulsory collaboration between interconnected network operators which are normally rivals in the Internet business marketplace [1].

In the literature, most of the existing research works on network virtualisation have been focusing on the realisation of virtualised platforms (including both communication [4] and automation [5]), optimisation algorithms for virtual node/link mapping and embedding [6-8], or application-specific virtual resource manipulations [9]. On the other hand, a systematic view on the fundamental interaction between SPs and NPs in holistically managing virtual network resources (VNRs) has been missing in the research community. In this article, we aim to shed light on how SPs and NPs should interact with each other for jointly manipulating VNRs from their own perspectives. It is worth 
mentioning that, due to the nature of heterogeneous services, their supporting VNRs comprise not only traditional network resources such as bandwidth capacity, but also other types of capabilities such as in-network storage space and computation intelligence. As shown in Figure 1, resource management in network virtualisation can be viewed into two distinct dimensions from the viewpoints of NPs and SPs respectively. Firstly, an NP needs to efficiently manage its own physical resources, which involves tasks such as logically partitioning physical resources into VNRs, and engineering them according to distinct class of service requirements. Upon receiving individual VNR requests from SPs, the NP considers the actual VNR slice allocation to each of them in a cost-efficient manner. As such, the actual physical resources owned by the NP are effectively shared between coexisting services with heterogeneous requirements. This dimension is known as vertical VNR management from the viewpoint of NPs. The other dimension is how an SP manages its rented VNRs which are used to form its own virtual network infrastructure for supporting the targeted services. As shown in the figure, an SP typically deploys its unified service platform across multiple autonomous NP networks, even including heterogeneous IP/MPLS backbone networks and mobile/wireless networks. In this case, it is the SP's responsibility to "concatenate" VNR capabilities provided from individual NPs to form a global virtual network infrastructure for supporting its own services to be deployed. This dimension is known as horizontal VNR management on the SP side.

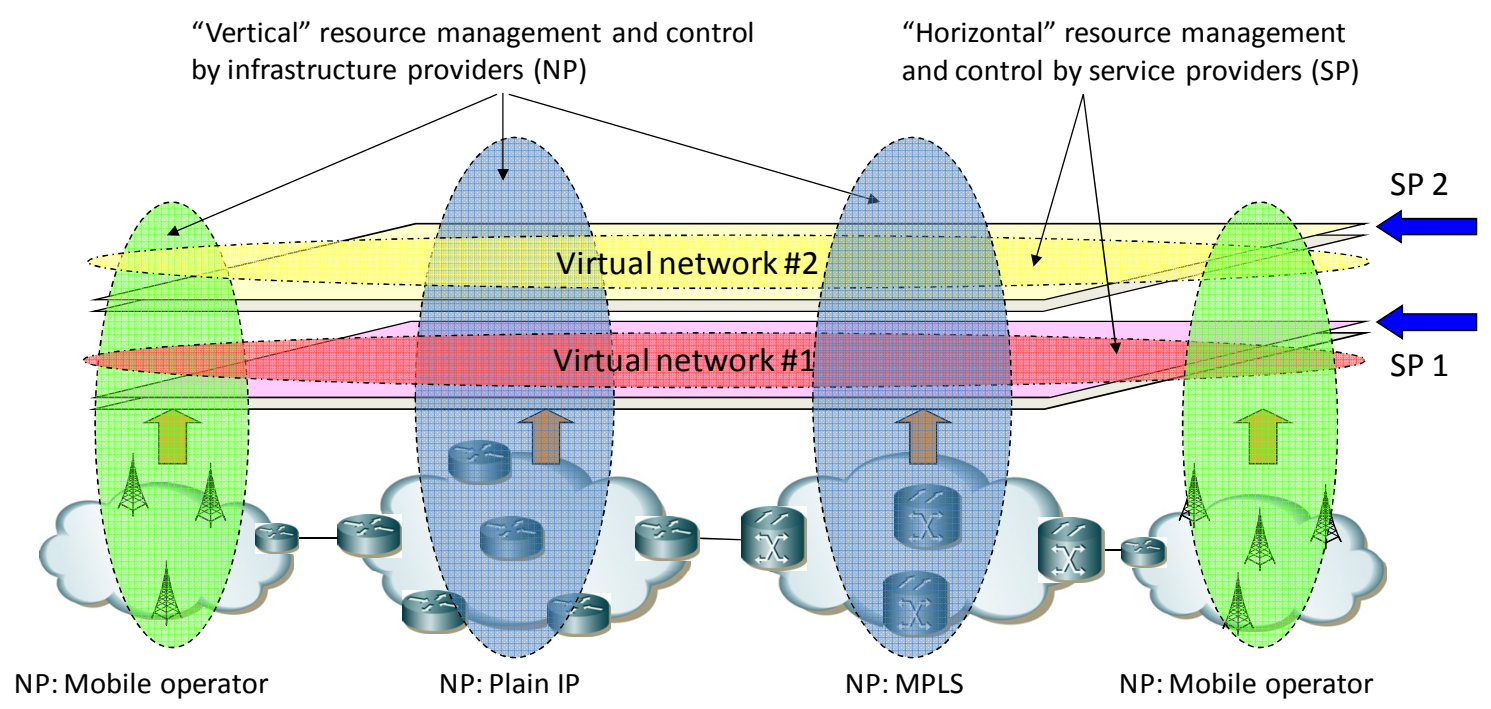

Figure 1. An illustration for two-dimensional virtual network resource management

In this article, we present a holistic architecture framework for VNR management according to the two-dimensional views from both the SP and the NP sides. A set of functional components and their interdependencies are specified for supporting VNR manipulation by the two types of stakeholders. With network virtualisation, SPs play a more active role in controlling network resources by means of deploying customised networking mechanisms and protocols dedicated to their own virtual network platforms. This is in contrast to the conventional scenario where heterogeneous SPs only demand specific service requirements, and passively rely on the underlying NP to dimension its physical resources accordingly, typically through unilateral traffic management/engineering paradigms [10][11][16]. Due to the fact that an NP normally treats customer traffic in an aggregated manner, the flexibility in providing service differentiation is hence limited. In this article we illustrate how SPs and NPs can systematically manipulate virtualised resources according to the two-dimensional management architecture in virtual network environments. A set of key VNR operations on both SP and NP sides are specified based on the proposed architecture and its functional components. 


\section{Architecture Overview}

Figures 2 and 3 respectively show the functional architecture for provisioning and controlling VNRs on the SP and NP sides. Fundamentally, an SP's role is to provide specific value-added services to customers based on the VNR "segments" rented from individual underlying NPs. In order to offer end-to-end services in the Internet, an SP may need to rent separate VNR segments from individual inter-connected NPs, and then horizontally "bind" them together to form an integrated virtual service platform on top of the physical network infrastructure. In this case, the top-level objective of SPs is to maximise their global service capability with minimum rented VNRs, typically through cost-efficient VNR provisioning and engineering operations. On the other hand, NPs rent their edge-to-edge VNR segments to incoming SPs with heterogeneous service requirements. It is the objective of NPs to attract and satisfy maximum incoming VNR requests based on available physical resources in order to maximise their revenues, typically through optimised provisioning and allocation of own network resources. It is worth mentioning that NPs, even backbone network operators, do not need to worry about the end-to-end service requirements on the SP side, as it is the responsibility of individual SPs to bind their rented VNR segments together according to their own service targets. The benefit is that, underlying network operators who are mainly rivals in the Internet marketplace, will not be forced to cooperate with each other in order to provide end-to-end services. Instead, each of them only care about how to appropriately allocate its own VNR segments to the requesting SPs, while each SP may apply its dedicated strategy in implementing the end-to-end service capability through its own VNR binding operation. A distinct example is for an SP to deploy its own routing protocol on top of its endto-end virtual network infrastructure with horizontally bound VNR capabilities which covers multiple underlying NP domains. In such a scenario, flexible and customised overlay routing on the SP side is able to override the rigid BGP routing configurations and path selections in the physical network.

\subsection{Horizontal VNR management on the SP side}

We first focus on the horizontal VNR management performed by SPs. First of all, an SP needs to plan its basic service objectives, for instance, what types of services to be offered to customers and what will be the service coverage in the Internet? Such Top-Level Service Planning function provides necessary guidance and policies to the VNR Provisioning and Binding component that is a decision maker for actual VNR requesting and engineering. VNR provisioning and binding plays the key role in resource management on the SP side at long timescale, e.g. monthly. Based on the forecasted customer demand that can be derived from service level agreements (SLAs) with customers, the function determines the appropriate VNRs to be rented from NPs in long-term operation. According to the expected service level objectives, this component also performs offline resource engineering based on the rented VNR segments provided from the underlying NPs, e.g. the determination of virtual node locations and virtual path selections. One distinct operation task in this case is VNR binding in order to concatenate local VNR capabilities rented from individual NPs for provisioning of a virtual platform with end-to-end service capabilities. Detailed description on VNR binding will be introduced in the next section.

A complementary function to long-term VNR provisioning is Adaptive VNR Control that caters for short-term or ad hoc VNR handling in dynamic environments. Specifically, due to potential unpredicted customer demands, statically rented VNRs might not be always sufficient for accommodating changing or new customer service requirements. In this case the Application Service Assurance Monitoring component needs to capture the up-to-date service conditions to the current end users on their ongoing applications. Such information needs to be fed into the Adaptive VNR Control component for on-the-fly decision making process, for instance, either to issue an adjusted VNR request to the NP based on the original (static) one, or simply to perform admission control over excessive incoming user demands.

A special case for VNR renting is the construction of short-term virtual network infrastructures for some one-off events. For instance, a media broadcasting service provider may want to establish a global content delivery network infrastructure based on information centric networking (ICN) technologies (e.g. the publish-subscribe paradigm) [12] in order to provide live broadcasting services for an event such as the Olympic Games or simply a music concert for a given period. In this case, 
there is no long-term VNR renting contract between this SP and the underlying NPs, and additionally the number of customers that will use such services might be very difficult to predict in advance. Under such circumstances, the media service provider may only request short-term virtual resources from the underlying NPs, and at the same time perform adaptive VNR control according to the actual user demand on the service. If necessary, adjusted VNR requests are negotiated for accommodating more incoming users, for instance in a flash crowd situation.

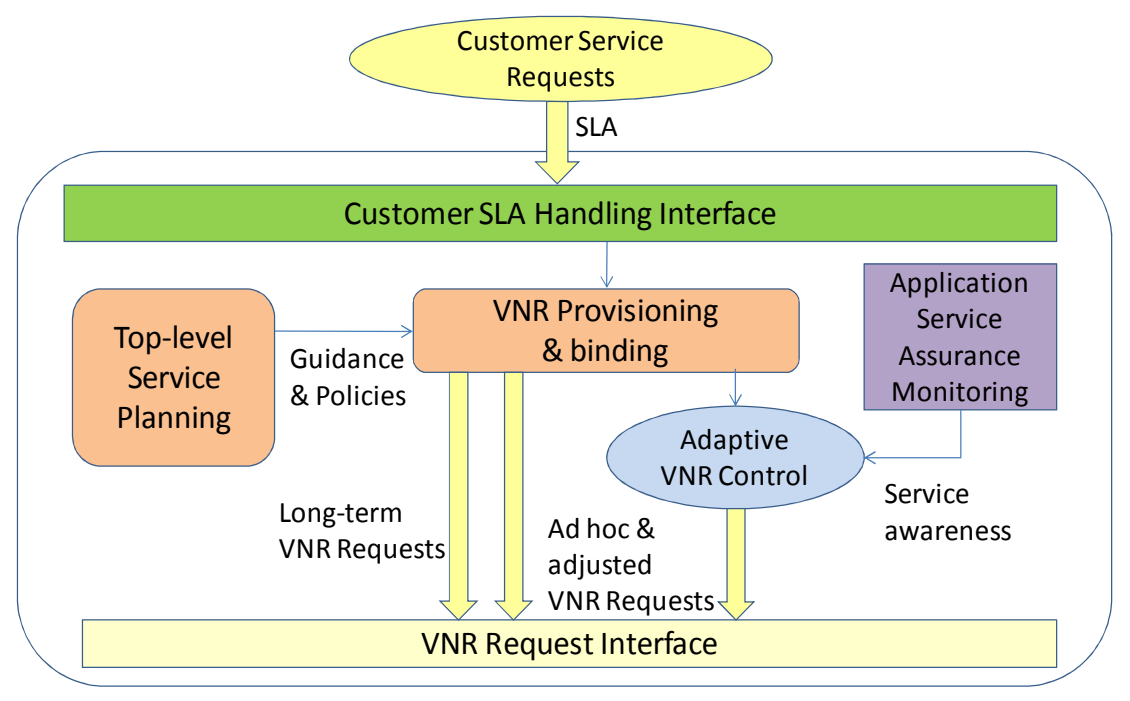

Figure 2. Functional architecture for horizontal VNR management

\subsection{Vertical VNR management on the NP side}

On the NP side, a key issue is how to partition its own network resources for optimised VNR engineering in order to maximise revenues with cost-efficient resource utilisations. For instance, if an NP is able to envisage that the majority of the requesting SPs will target multimedia-based content delivery services, then the provisioning of the VNRs should take into account the common quality of services (QoS) requirements such as bounded edge-to-edge delay and bandwidth support, and possibly even content caching capabilities. On the other hand, given the uncertainty in the VNR renting modes from SPs (e.g. long-term vs. short term / ad hoc), it is also beneficial for an NP to have initial planning in dealing with different types of incoming VNR requests, for instance determination on the proportion of VNRs reserved only for ad hoc or short-term requests. These issues are to be considered by the Top-Level VNR Planning component which provides general guidance and policies to the VNR Engineering component for handling specific VNR requests. An example of such guidance can be that, the maximum edge-to-edge delay associated with any VNR segment allocated to SPs should be 100 milliseconds, as this upper bound is the outcome of the dimensioning of physical network resources.

Upon receiving a specific VNR request from an SP, the NP start to process it through the VNR Request Handling Interface component which provides necessary input to the central VNR Engineering component. The VNR Engineering component plays an essential role in decision-making process during network operations. Normally, long-term VNR requests should be processed in batches together at certain timescale, e.g. at the interval of every month. Once these requests have been accepted, the corresponding VNR segments are actually allocated through the Offline VNR Allocation operation. The benefit of batch-based VNR request processing is that such an operation gives the NP a clear view on the global VNR availability at the top level in order to perform long-term VNR allocations. As mentioned previously, due to the uncertainty associated with the service demand requested by end users on the SP side, an SP may issue adjusted VNR requests on the fly based on 
their original long-term VNR requests. On the other hand, according to the guidance provided from the VNR Planning component, a specific portion of VNR capabilities can be reserved for the allocation upon some ad hoc VNR requests at any time. To deal with both adjusted VNR requests and brand new ad hoc VNR requests, the short-term adaptation intelligence in VNR engineering is needed for making run-time decisions on whether or not to accept them. Such decisions are typically made based on necessary inputs from both service and network monitoring functions that are responsible for reporting the up-to-date service and resource utilisation conditions. As the outcome, the Online VNR Allocation component is responsible for enforcing the actual on-the-fly VNR allocations.

The VNR Service Assurance Monitoring component is responsible for verifying the fulfilment of the current VNR allocations, while the Network Resource Monitoring component provides complementary information on the current network conditions and physical resource availability. For instance, in event of network anomaly in the physical network (e.g. link or node failure), it is the responsibility of the network resource monitoring function to immediately inform the $V N R$ Engineering component for taking necessary recovery actions [13]. Depending on the severity of the fault, short-term adaptations can be applied for performing local service recovery for the affected VNR segments without disrupting the on-going service sessions. In case of a serious or large scale network failure, then the VNR Engineering component may need to interrupt all on-going VNR sessions and perform global network recovery in an offline manner.

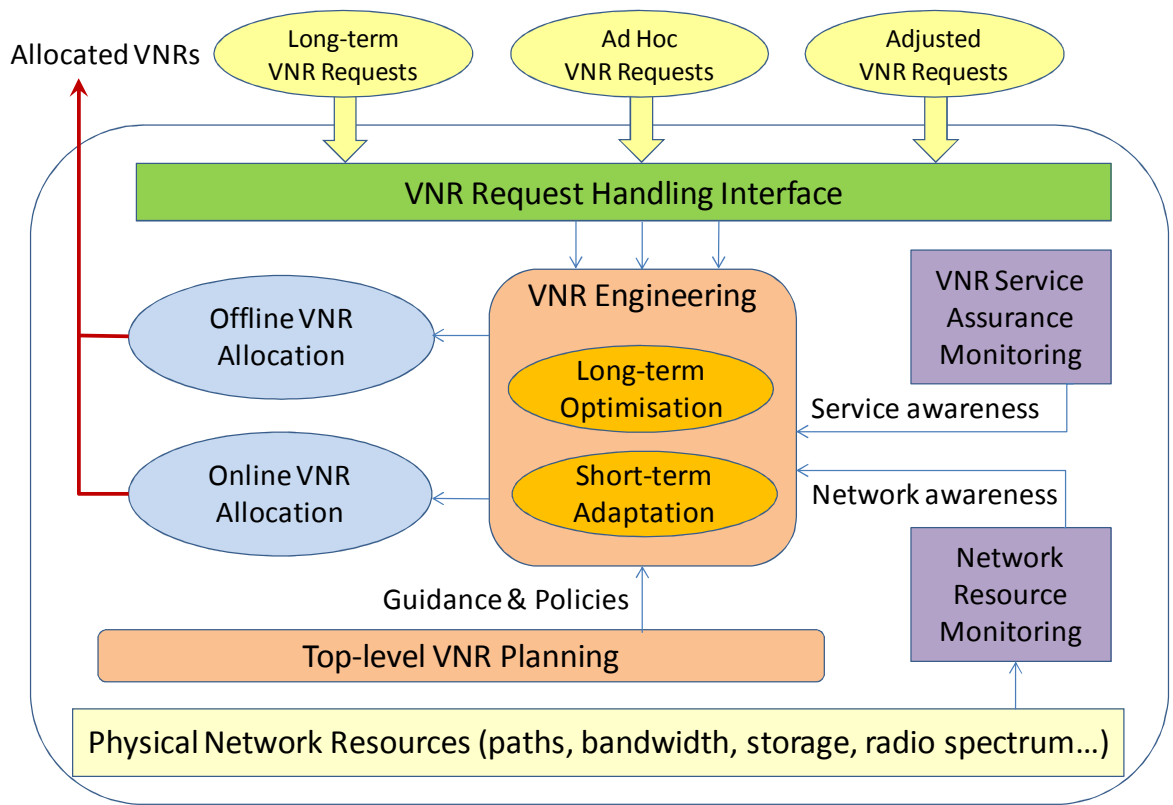

Figure 3. Functional architecture for vertical VNR management architecture

\section{Discussion on Key VNR Operations}

In this section we discuss in detail the key VNR operations in the two dimensions of the VNR management paradigm, namely VNR requesting and binding on the SP side and VNR allocation on the NP side.

\subsection{VNR requesting}

According to heterogeneous service characteristics on the SP side, the corresponding VNR requests may have very different requirements on the virtual resources to be rented. We first summarise below 
a set of common properties that are generally needed in all types of VNR requests. Following that, we illustrate two distinct examples of heterogeneous VNR requesting based on different types of services.

In general, the following information is necessary to be expressed in all types of VNR requests. From the functional architecture point of view, unless specifically indicated, it is the Top-Level Service Planning component on the SP side that should determine this set of generic properties.

- VNR coverage and topology. Fundamentally, an SP should specify in its VNR request the targeted geographical service coverage on top of the physical network infrastructure. This can be the case when the underlying NP has a multi-continental Internet access coverage, while the requesting SP only deploys its service within a specific country or continent. In addition, the topology of the virtual network infrastructure should also be specified, for instance the placement of virtual nodes and establishment of virtual links, optionally with dedicated capability support (e.g. storage, computing) to be associated with the virtual nodes and dedicated bandwidth support to be associated with the virtual links in the infrastructure. The actual optimisation of the virtual network topology by taking into account specific customer demands is covered by the VNR Provisioning and Binding function component.

- VNR renting timescale. In all VNR requests, the timescale of planned resource renting should also be specified. Certainly this is obvious on the SP side, depending on how long the deployed services are to be provided to end users. Concerning NPs, as previously mentioned, long-term VNR requests not only help the underlying NPs to efficiently provision its VNRs to be allocated, but also they are beneficial to network stability during operation period. It is not difficult to infer that, having excessive ad hoc or short-term VNR renting requests makes it difficult for NPs to efficiently manage its network resources to be shared.

- Runtime VNR adjustment options. Due to unpredicted service subscription demand from end users, an SP may need to adjust its VNR requests during runtime if necessary. In order to avoid potential contentions between SPs on VNRs requesting adjustments during network operation time, each $\mathrm{SP}$ and the NP can negotiate a priori in the initial VNR request on how future runtime request adjustments can be performed. One scenario is to specify an upper limit of additional VNR capabilities that can be requested in any future runtime adjustment from the SP. In case the additionally requested VNRs are still not sufficient for supporting incoming end users, then the SP needs to apply its own admission control policies to avoid service disruptions to existing end users.

- Charging/tariff model. This simply indicates how SPs' usage of the VNRs will be charged by NPs. The price of the VNRs may depend on not only the types and locations of the resources to be requested but also the time (peak/off-peak) at which the NP is operating.

Now we use two examples to illustrate customised VNR requesting scenarios in supporting different types of services. We consider a Voice over IP (VoIP) service provider (VSP), and a content service provider (CSP) that offers Video-on-Demand services based on ICN technologies with various innetwork content manipulating functions. Table 1 below summarises some key differences concerned by the VSP and the CSP when they make VNR requests for deploying their own services. For instance, while a VSP only requires the virtual communication infrastructure for supporting end-to-end IP telephony services, a CSP may need more comprehensive in-network virtual resources such as distributed content storage space and in-network computing intelligence for on-the-fly adaptation of video content while it is being transmitted towards end users with different terminal capabilities. On the other hand, interactive communications in VoIP applications demand low delay/jitter, while bandwidth availability plays a more important role in multimedia content distribution services. 


\begin{tabular}{|c|c|c|}
\hline & VNRs concerned by VSP & VNRs concerned by CSP \\
\hline Types of VNRs & $\begin{array}{l}\text { - Only virtual communication } \\
\text { infrastructure ( including } \\
\text { virtual nodes/links/paths) in } \\
\text { most cases }\end{array}$ & $\begin{array}{l}\text { - Virtual communication } \\
\text { infrastructure } \\
\text { - Virtual in-network content } \\
\text { storage capability } \\
\text { - Virtual in-network content } \\
\text { adaptation capability } \\
\end{array}$ \\
\hline $\begin{array}{l}\text { VNR QoS } \\
\text { requirements }\end{array}$ & $\begin{array}{ll}- & \begin{array}{l}\text { Bounded edge-to-edge delay } \\
(<150 \text { milliseconds) and jitter }\end{array} \\
\text { - } & \text { Bidirectional QoS } \\
\text { requirements for supporting } \\
\text { interactive VoIP services }\end{array}$ & $\begin{array}{l}\text { - Stringent bandwidth support for } \\
\text { video content delivery } \\
\text { - Mainly QoS assurance in the } \\
\text { direction from content sources to } \\
\text { individual consumers }\end{array}$ \\
\hline $\begin{array}{l}\text { VNR reliability } \\
\text { requirements }\end{array}$ & $\begin{array}{l}\text { - Stringent demand on } \\
\text { communication network } \\
\text { reliability } \\
\text { - Five 9's availability for } \\
\text { assuring end-to-end } \\
\text { conversations }\end{array}$ & $\begin{array}{l}\text { - Stringent demand on } \\
\text { communication network } \\
\text { reliability } \\
\text { - More tolerable on storage } \\
\text { reliability due to availability of } \\
\text { multiple content replicas }\end{array}$ \\
\hline
\end{tabular}

Table 1. VNR renting concerns by different types of SPs with heterogeneous requirements

\subsection{VNR binding}

VNR binding refers to SPs' concatenation operations on individual VNR segments rented from multiple interconnected NPs in order to provision specific end-to-end services. Such operation should be fulfilled by the VNR Provisioning and Binding function component. Figure 4(a) shows a simple example on horizontal virtual resource binding by a CSP whose service coverage spans two underlying NP networks. In this example both NP 1 and NP 2 allocate their own VNR substrates to the CSP upon its request. It is worth mentioning that these two substrates are edge-to-edge virtual resource segments bounded within each NP's physical network. In this case, it is the responsibility for the CSP to deploy its own mechanisms to concatenate the two VNR segments together in order to have an end-to-end content delivery infrastructure. One typical example for such a binding is to enforce CSP's own end-to-end content routing protocol across the unified virtual platform. By means of efficient VNR binding, end-to-end QoS can be engineered on the SP side for supporting corresponding services.

Take Figure 4(a) as an example, if we assume that the edge-to-edge VNR delay offered from the two NPs is 50 milliseconds each, the CSP may enforce its own path selection on top of the global virtual platform and determine the targeted end-to-end delay performance, say 100 milliseconds. It is worth mentioning that the outcome of the VNR binding operations might not be always simply the "sum" of the concatenated VNR capabilities from individual NPs. Specifically, SPs have high degree of flexibility in enforcing their own routing or forwarding policies to partially override the routing/forwarding decisions in the underlying network. For instance, if an SP needs to achieve loadbalancing over its own virtual network platform with dedicated bandwidth allocation from the NPs, it may apply intelligent virtual traffic engineering (TE) schemes, e.g. to avoid local congestion by enforcing possibly longer virtual paths as compared to the direct concatenation of underlay paths. Mobility support across multiple administrative networks is another aspect to be considered in VNR binding operations. For instance, when an end user is consuming a live content while moving from one physical NP to another (e.g. across two mobile operator networks), the end-to-end virtual network platform should make sure the service is not disrupted when the user is moving across network boundaries. 


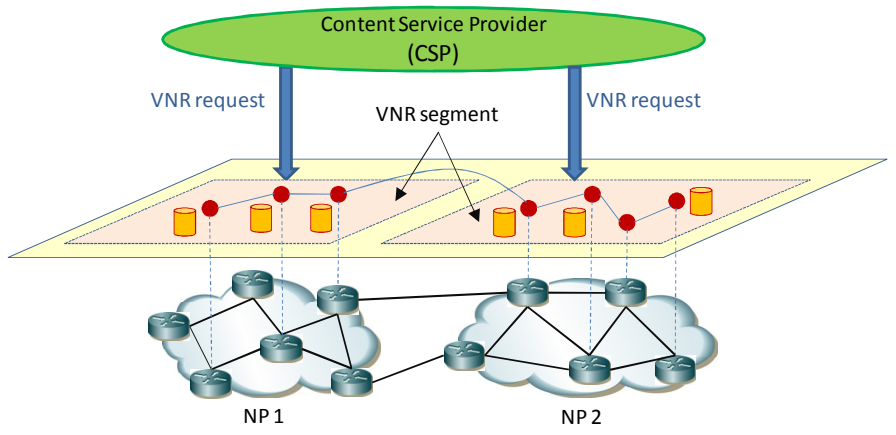

(a) Horizontal VNR binding by $S P$

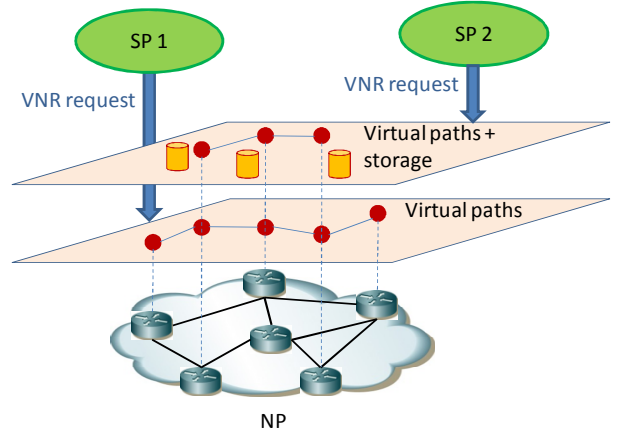

(b) Vertical VNR allocation by NP

Figure 4. VNR binding and VNR allocation

\subsection{VNR allocation}

From the viewpoint of NPs, the ultimate goal of VNR allocation is to achieve maximum VNR service capability for maximising their revenues while maintaining optimised network performances. Given the existence of heterogeneous SPs that cover a wide variety of Internet-based services, a key challenge faced by NPs is how to systematically provision different types of VNRs (bandwidth, spectrum, in-network storage etc.) for supporting all of them. As shown in Figure 4(b), SP 1 only requires virtual communication infrastructure (e.g., virtual paths), while SP2 also needs other types of VNRs such as virtual network storage. This requires the underlying NP to appropriately provision diverse VNRs accordingly for coping with different types of requests.

Given the statically provisioned physical resources (e.g. bandwidth capacity of physical network links, actual memory space available at network elements), it is NPs' responsibility to make sure they are allocated to requesting SPs in a cost-efficient manner. For instance, from Figure 4(b) we can also see that a physical resource (e.g. a router or a network link) needs to be virtualised and allocated to multiple sharing SPs. A key issue to be concerned by the underlying NP (more specifically the VNR Engineering function component) is how to achieve balanced VNR allocations across the entire network infrastructure such that the risk of having VNR availability bottlenecks is minimised. In this context, we envisage two specific technical challenges in achieving this. First, high degree of on-thefly VNR request adjustments by SPs incurs uncertainty from the perspective of virtual resource availability seen by the NP during allocation. Secondly, many real-time applications demand stringent network robustness, e.g. sub 50-millisecond post-failure disruption time according to common practice. Certainly, the corresponding protection function should be implicitly embedded in the VNRs before they are allocated to SPs, rather than relying on SPs to deploy their own application-level protection mechanisms which suffer from slow reaction to network-layer failure events. As such, if an NP embeds network-layer failure protection functions in VNRs to be allocated, it needs to take into account additional backup resource capabilities (e.g. alternative fast reroute [13] paths with bandwidth support) to be activated upon failures. It is certainly desirable to have shared backup resources that are able to simultaneously provide protections to the affected VNRs requested by multiple SPs [14].

\section{Summary and Future Research Directions}

In this article we have presented a holistic management architecture for providing necessary guidance to virtual network resource management on both the SP side and the NP side. The proposed twodimensional framework sheds light on how virtual network resources should be collaboratively managed by distinct stakeholders in the Internet business market. From the SP's point of view, the key issue is to how to optimally rent virtualised resources and horizontally bind them together in order to form an end-to-end service platform for supporting the deployed services. On the other hand, NPs are responsible for independently managing own network resources, logically partitioning them and allocating to heterogeneous SP providers. Such vertical resource management at the NP side offers a 
highly flexible solution to the provisioning of physical resources for supporting a wide variety of networked services on top of the physical network infrastructure.

As our future work, we will validate the proposed two-dimensional architecture for VNR management on top of realistic virtual network platforms such as Dragon-Lab (AS number 24575) [15] which offers an ideal experimental environment at the size of a real NP network. Meanwhile, we have also envisaged a set of future research directions concerning VNR management at both the SP and NP side. From the viewpoint of SPs, a general open issue is how to holistically provision customised VNR platforms in a cost-efficient manner, mainly through joint optimisation in VNR renting, virtual topology control and end-to-end service delivery path control. For VNR provisioning by CSPs, it is also interesting to investigate how content caching can be optimally achieved within their virtual network platforms. On the NP side, flexibility in VNR allocation to heterogeneous requesting SPs is an essential issue to be concerned. "Soft" VNR allocation strategies are desired, in which case VNR capabilities (e.g. bandwidth support) are not statically allocated, but instead coexisting SP virtual networks can adaptively share some common network capabilities in dynamic environments. Energy efficiency on the NP side will be another research issue to be investigated. The objective is to strategically reduce the physical network capability (through link/node sleeping or rate adaptation) when the end user demand on top of the SP virtual networks are relatively low, for instance during off-peak time period. In this case, the underlying NP may optimally perform sleeping or rate adaptation configurations for energy saving, but without disrupting the service performance on the SP side. Last but not least, although security aspects have not been particularly discussed in this article, it is certainly a vital issue to be addressed given the open virtual environment that allows resource sharing between different parties/applications [17]. It will be certainly our future research work to consider security and access control mechanisms on the NP side against illegitimate access of network resources and potential denial of service attacks from malicious entities.

Acknowledgement

This work is partially funded by the EU FP7 IRSES EVANS project (Grant Agreement Number: PIRSES-GA-2010-269323)

\section{References}

[1] N. Feamster, J. Rexford and L. Gao, "How to Lease the Internet in Your Spare Time", ACM Computer Communication Review, January 2007

[2] M. Yu, Y. Yi, J. Rexford, and M. Chiang, "Rethinking virtual network embedding: Substrate support for path splitting and migration", ACM SIGCOMM Computer Communications Review, April 2008

[3] M. Boucadair, P. Georgatsos, N. Wang, D. Griffin, G. Pavlou, M. Howarth and A. Elizondo, "The AGAVE Approach for Network Virtualisation: Differentiated Services Delivery", Annals of Telecommunications, Vol. 64, No. 5-6, pp. 277-288, 2009

[4] M. Chowdhury, R. Boutaba, "A Survey of Network Virtualization", Elsevier Computer Networks, Vol. 54(5), pp. 862-876, April 2010.

[5] J. Beran, P. Fielder and Z. Zezulka, "Virtual Automation Networks", IEEE Industrial Electronics Magazine, Vol. 4, Issue 3, 2010, pp. 20-27

[6] M. Chowdhury, M. R. Rahman and R. Boutaba, "ViNEYard: Virtual Network Embedding Algorithms with Coordinated Node and Link Mapping", IEEE/ACM Transactions on Networking, Vol. 20, Issue 1, 2012, pp. 206-219

[7] Y. Zhu and M. Ammar, "Algorithms for Assigning Substrate Network Resources to Virtual Network Components", Proc. IEEE INFOCOM 2006 
[8] I. Houidi, W. Louati and D. Zeghlache, “A Distributed Virtual Network Mapping Algorithm”, Proc. IEEE ICC 2008

[9] Y. Wang, Y. Jin, W. Guo, W. Sun and W. Hu, "Virtualized Optical Network Services Across Multiple Domains for Grid Applications", IEEE Communication Magazine, Vol. 49, No. 5, 2011

[10] K. Xu, H. Liu, J. Liu, J. Zhang, "LBMP: A Logorithm-Barrier-Based Multipath Protocol for Internet Traffic Management", IEEE Transactions on Parallel and Distributed Systems, Vol. 22. No. 3, 2011

[11] N. Wang, K. H. Ho, G. Pavlou and M. Howarth, "An Overview of Routing Optimization for Internet Traffic Engineering", IEEE Communications Survey and Tutorial, Vol. 10, No. 1, 2008

[12] P. Jokela, A. Zahemszky, C. E. Rothenberg, S. Arianfar and P. Nikander, "LIPSIN: Line Speed Publish/Subscribe Inter-networking", in Proc. ACM SIGCOMM '09, Barcelona, Spain, August 2009

[13] M. Shand and S. Bryant, "IP Fast Reroute Framework", IETF RFC 5714, January 2010

[14] T. Guo, N. Wang, K. Moessner, and R. Tafazolli, "Shared Backup Network Provision for Virtual Network Embedding", Proc. ICC 2011

[15] J. Wang, Z. Li, G. Lv, C. Jiang, X. Li and Q. Zhang, "Dragon-Lab: Next Generation Internet Technology Experimental Platform", Science in China Series F: Information Sciences, Vol. 51, No. 11, 2008, pp. 1908-1918

[16] S. Shin and M. Yoon, "Virtual vectors and network traffic analysis", IEEE Network, Vol. 26, Issue 1, 2012, pp. 22-26

[17] L. Zhou and H. Chao, "Multimedia traffic security architecture for the internet of things", IEEE Network, Vol. 25, Issue 3, 2011

\section{Biographies}

NING WANG (n.wang@surrey.ac.uk) is a lecturer at the Centre for Communication Systems Research (CCSR), University of Surrey in UK. He received his B.Eng. (Honours) degree from Changchun University of Science and Technology, P.R. China, in 1996, his M.Eng. degree from Nanyang Technological University, Singapore, in 2000 , and his Ph.D. degree from the University of Surrey in 2004. He is the technical coordinator of the EVANS project. His research interests include virtual and overlay networks, information centric networking and QoS/Energy aware network optimizations.

YAN ZHANG (yanzhang@ieee.org) received a Ph.D. degree from Nanyang Technological University, Singapore. He is working with Simula Research Laboratory, Norway; and he is an adjunct Associate Professor at the University of Oslo, Norway. He is an associate editor or guest editor of a number of international journals. He serves as organizing committee chairs for many international conferences. His current research interests include wireless networks, cognitive radio and smart grid.

JOAN SERRAT-FEMANDEZ (serrat@tsc.upc.edu) received the degree of Telecommunication Engineer in 1977, and the Doctor degree in Telecommunication Engineering in 1983, both from Universitat Politècnica de Catalunya -UPC-. Currently he is Full Professor at UPC where he has been involved in several collaborative projects with different European research groups, both through bilateral agreements or through participation in European funded projects. His topics of interest are in the field of autonomic network and service management.

JUAN-LUIS GORRICHO (juanluis@entel.upc.edu) is Associate Professor at the Polytechnic University of Catalonia (UPC) in Barcelona, Spain. He received his M.S. degree in Telecommunications Engineering in 1994 from the UPC, and his Ph.D. degree in 1999 from the same University. He is an active member of the Management, Pricing and Services for Next Generation Networks research group (MAPS) from the Telematics Department of the UPC. Presently he is focusing his research in the fields of network management, context-awareness and reinforcement-learning agents. 
TAO GUO (t.guo@surrey.ac.uk) received a PhD degree in Wireless Networks from Newcastle University, UK, in 2009. He is currently a research fellow in Centre for Communication Systems Research, University of Surrey, UK. He has been working on various UK EPSRC and EU FP7 projects such as BeFEMTO, QoSMOS, and Mobile VCE Core 5 "Flexible Networks". His current research interests include mobility and radio resource management, and network virtualization.

ZHENG HU (huzheng@bupt.edu.cn) received Ph.D. Degree in 2008 from Beijing University of Posts and Telecommunications (BUPT). Currently, he is working with University of Posts and Telecommunications, and his research interests lie in the next generation wireless network and services focusing on resource management and network control. He is also coordinating and participating several research projects such as Chinese Key program, 973 program, and 863 program.

PING ZHANG (pzh@bupt.edu.cn) received Ph.D. degree in 1990 from Beijing University of Posts and Telecommunications (BUPT). Currently, he is a Professor in BUPT and the Director of Key Laboratory of Universal Wireless Communications, Ministry of Education. He is Executive Associate Editor-in-chief on information sciences of Chinese Science Bulletin, a Member of next-generation broadband wireless communication network in National Science and Technology Major Project committee, a Member of the 5th Advisory Committee of NSFC(National Natural Science Foundation of China), the Chief Scientist of "973" National Basic Research Program of China. His current research interests include wireless communication, cognitive radio networks, and next generation wireless network. 